\title{
Magnetoelectric effects theory by Heisenberg method based on permutation group symmetry of nanoparticles
}

\author{
S. Leble \\ Immanuel Kant Baltic Federal University, Institute of Physics, \\ Mathematics and Informational Technology, Russia, Kaliningrad 236000 \\ lebleu@mail.ru
}

PACS 75.70.Cn, 05.30.d, 02.20.a

DOI 10.17586/2220-8054-2020-11-1-50-64

\begin{abstract}
The Heisenberg theory of ferromagnetism is widened to include external electric field action. The material relations are derived by means of differentiation of logarithm of partition function with respect to the magnetic and electric fields. The mean energy coefficients as the exchange integrals combinations are expressed via characters of irreducible representations of corresponding permutation groups by the Heitler method. The thermodynamic equations of state for polarization and magnetization, as functions of the electric and magnetic fields, are derived and illustrated by figures. The magnetization and hysteresis curves in magnetization - magnetic field components plane are built. The theory is applied to nanoparticles, the particle partition function is modeled as the product of the surface and bulk parts. The statistical sum is constructed having explicit expressions for the mean energy in terms of exchange integrals and number of closest neighbors for surface and bulk atoms. The relative contribution of the surface and bulk terms is evaluated.
\end{abstract}

Keywords: multielectron states, permutation group symmetry, mean energy, Gauss distribution, Heisenberg chain, electric field, nanoparticles, multiferroics.

Received: 20 January 2020

Revised: 10 February 2020

\section{Introduction}

\subsection{General remarks}

An exchange integral for electron pair interaction enters the theory of magnetic phenomena as a basic notion from times of pioneer paper of Heisenberg [1]. In majority of papers on magnetic phenomena applications it is used as a unique parameter, conventionally established by experiments for a given class of matter [2]. A main result of the Heisenberg work is the relation that includes the matter parameters, temperature and magnetic field. It contains information of the distribution of atoms in space via number of closest neighbors $z$. This relation is an transcendental equation for the magnetization vector component, that, dependent on the matter parameters values, has one or two solutions. The last is identified with ferro-magnetism existence. Its estimation by the Heitler-London model approximation, as it is made in [1] can be improved by Fockian eigenfunctions [3], which, however, is a complicated numerical task.

This seminal paper [1] theory exhibits the next important ingredient of the multi-electrons' system natural symmetry, with respect to the permutation group. Following [4], it uses evaluation of mean energy for a given irreducible representation of this group, expressing it explicitly via exchange integrals. On same platform, explicit formulas for mean quadratic deviations of the energy is derived by Heisenberg [1] and used for energy distribution modeling in Gaussian form. Some natural critics of this theory is published in [5] with respect to its applicability to the case of 8 nearest neighbors case $(\mathrm{Fe})$, but exhibits a good physical realization for a 12 neighbors matter $(\mathrm{Ni}, \mathrm{Co})$, illustrated by comparison with experiments $[6,7]$.

The $a b$ initio models such as Brillouin or Langevin ones developed by Weiss either in a framework of molecular theory, or in combination with the phenomenology of Landau, improves paramagnetic domain description for a ferromagnetic matter, but not so good in a hysteresis curves production [8,9]. It also do not take into account the fundamental permutation group symmetry.

In [10] Nakano represents the results with the lower (compared to Heisenbergs') minimal number of closest neighbors $z=6$, that opens a possibility of using this model for Iron. Next, W. Heisenberg [11] develops the theory with the domain walls account. The whole story of the ferromagnetism theory, related to Heisenberg contributions, is outlined in [12].

According to Kondorsky classification [13], there are three main causes of hysteresis: 1) Hysteresis due to a delay in the displacement of boundaries between domains. 2) Hysteresis due to growth retardation of the magnetization reversal of nucleation. 3) Hysteresis due to irreversible rotation. 
It is known that the continuous version of Heisenberg chain equation with Gilbert term account [14] may explain existence of domain walls (DW). An energy of a DW and conditions of its creation may be estimated in terms of exchange integral and anisotropy coefficient. The first is proportional to the so-called exchange stiffness and the second is related to a distribution of atoms in space [15].

The hysteresis curve for nanocrystallized soft magnetic systems was extracted from the intrinsic switching field distribution of the sample, as it is simulated with a combination of a Gaussian distributions [16], that has an intersection of ideas with [11]; other approach is based on the solid base of Landau-Lifshitz-Gilbert equations [17].

The model we presented recently is a generalization of Heitler-Heisenberg method of energy mean values and mean quadratic deviations of the energy derivation by explicit formulas via characters of irreducible representation of permutation group. The generalizations widen the symmetry group including space transformation and external electric field [3]. As a consequence of a difference between the nearest neighbors relative distance, fixed by positions in an atomic net, two kinds of the exchange integrals were introduced.

In this work, going down with dimension of objects under consideration from macroscopic to mesoscopic such as "fine particles" or "nanoparticles", we should take into account a relative weight of surface atoms in the whole object description [18]. There is important direction of the Barium titanate (BTO) nanoparticles investigations [19] related to the ferroelectricity phenomenon, known from times of [20].

The magnetoelectric effect has tremendous potential applications. Transition metal oxides provide a fertile playground for such phenomena. First-principles methods to study magnetoelectric phenomena are reviewed in the present text.

The search for materials displaying a large magnetoelectric effect has occupied researchers for many decades [21]. The rewards could include not only advanced electronics technologies, but also fundamental insights concerning the dielectric and magnetic properties of condensed matter. In this article, we focus on the magnetoelectric effect (e.g. in transition metal oxides) and review the manner in which first-principles calculations have helped guide the search for (and increasingly, predicted) new materials and shed light on the microscopic mechanisms responsible for magnetoelectric phenomena [22].

In this paper, we plan to go by the second way, introducing three kinds of the parameters (integrals) that link atoms as 1) bulk-bulk; 2) surface-surface; 3) bulk-surface.

The Heisenberg sum by atoms in such division would be shared to three subsums, with corresponding factors $J_{1}, J_{2}, J_{3}$. The statistical sum is correspondingly factorized. In this note, to show the principle, we restrict ourselves by two terms, unifying the last two parts, deriving and illustrating matter relations, including hysteresis curves for the simplest bulk case and bulk-surface.

\subsection{Notations. Partition function. Thermodynamics}

Let a Hamiltonian $\hat{H}$ be a function of external thermodynamic parameter $a$, which is conjugate to the internal parameter $B$, so that the elementary work is equal to $B d a$. Within the framework of the equilibrium quantum statistical physics, for given Boltzmann constant $k$ and temperature $T$, the Gibbs operator [23]

$$
\hat{f}=\frac{1}{Z} \exp \left[-\frac{\hat{H}}{k T}\right],
$$

contains the normalization constant,

$$
Z=\operatorname{Tr}\left[\exp \left[\frac{-\hat{H}}{k T}\right]\right]
$$

called a statistical sum (partition function). Then

$$
B=-k T \frac{\partial \ln Z}{\partial a}
$$

In this text we shall consider two pairs of the parameters: $a, B \rightarrow H, M ; E, P$, these are the component of magnetic field versus component of magnetization vector; the component of electric field versus component of polarization vector. For the sake of clarity, we act in a projection of all fields to one direction, so the Hamiltonian is a function of the magnetic and electric fields components along the marked direction $\hat{H}=\hat{H}(H, E)$. If the Hamiltonian is divided in few terms as $\hat{H}=\hat{H}_{1}+\hat{H}_{2}$, the partition function is factorized as $Z=Z_{1} Z_{2}$, hence the thermodynamic variable

$$
-\frac{B}{k T}=\frac{\partial \ln Z_{1} Z_{2}}{\partial a}=\frac{\partial \ln Z_{1}}{\partial a}+\frac{\partial \ln Z_{2}}{\partial a}
$$

correspondingly splits. 


\section{Heisenberg relation and hysteresis existence condition}

\subsection{Origin of the Heisenberg relation}

We start with the bulk case. After transformations, described in [24] (some explanations are given in subsequent sections) the partition function $Z$, defined by (1), is the following function of most probable magnetization $M$ per electron:

$$
Z=F\left[2 \cosh \frac{\omega}{2}\right]^{2 n}
$$

where:

$$
\omega=\alpha+\beta M-\beta^{2} \frac{M}{z}+\beta^{2} \frac{M^{3}}{2 z}, \quad \alpha=\frac{e \hbar}{m k T} H, \quad \beta=\frac{z J}{k T},
$$

$z$ - number of closest neighbors, $e, m$ - electron charge and mass, $n$ - number of electrons, $\hbar$ - Plank constant, $J-$ exchange integral.

The value of magnetization as thermodynamic variable is determined by (2) and (4) as [23]:

$$
M=\frac{1}{n} \frac{\partial \ln Z}{\partial \alpha}=\tanh \frac{\omega}{2} .
$$

Finally, the Heisenberg relation appears as:

$$
M=\tanh \frac{\alpha+\left(1-\frac{\beta}{z}\right) \beta M+\beta^{2} \frac{M^{3}}{2 z}}{2},
$$

the 1.h.s. of (7) we would note as $y_{1}(M)=M$ and the r.h.s. as $y_{2}(M, \alpha)$.

\subsection{On Heisenberg relation solution}

The number of intersections of the straight line $y_{1}=M$ and the curve $y_{2}=y_{2}(M, \alpha)$ for given $\alpha$ depends on the curve inclination in the origin. Let us illustrate it by plots, taking the closest neighbors number $z=12$ as for nickel and cobalt. At the Fig. 1 plots of the $y_{1}$ and three curves $y_{2}$ with different values of $\alpha$ are shown for the paramagnetic case $\beta=1$.

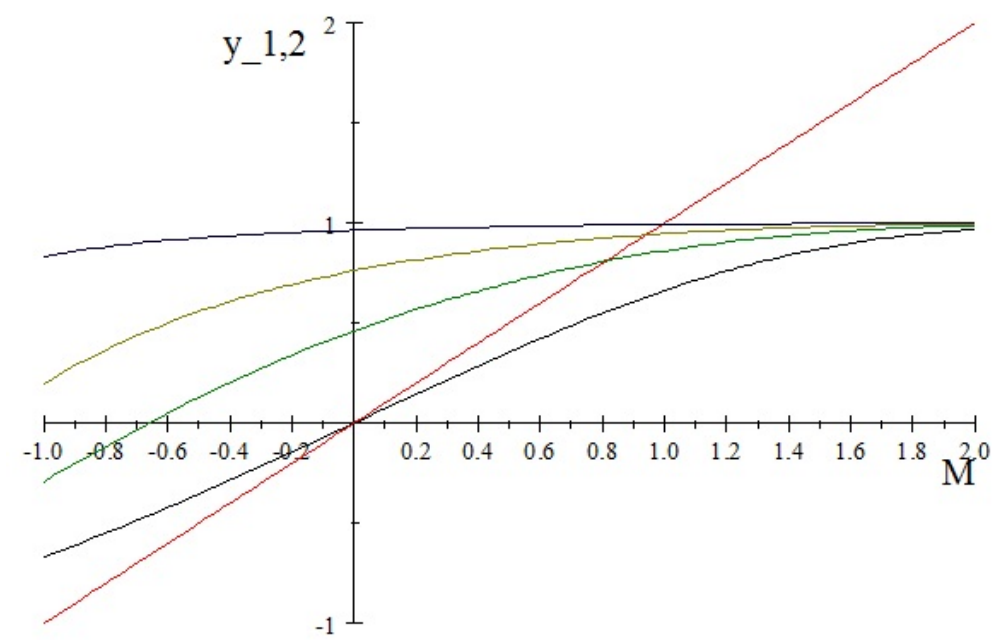

FIG. 1. $\beta=1$. The straight line (red) is $y_{1}=\mathrm{M}$. The curves from lower to upper $y_{2}(M, 0)-$ black $4, y_{2}(M, 0.5)$ - green $-3, y_{2}(M, 1.0)$ - brown $-2, y_{2}(M, 2.0)$ - navy - 1, with numbers at left

The intersection points for a sequence of unique values of $\alpha$ (proportional to the magnetic field, see (5)) lie on the magnetization curve, the phenomenon of saturation is quite visible. So, the intersection points form the magnetization curve. 


\subsection{Critical curve of ferromagnetizm existence}

As it is seen from the graphical view, for the ferro-magnetism in the present condition, the curve $y_{2}(M, \alpha)$ inclination at $M=0$ is less than one for $y_{1}$, or

$$
\beta\left(1-\frac{\beta}{z}\right) \geq 2
$$

At critical point of transition from unique to double solution, the inclinations at origin for both $y_{1,2}$ coincide, whence the derivative of the function $y_{2}(M, 0)$ should be equal to 1 :

$$
\left.\frac{\partial y_{2}(M, 0)}{\partial M}\right|_{M=0}=\frac{1}{2}\left(1-\frac{\beta}{z}\right) \beta=z \frac{J}{2 k T}\left(1-\frac{J}{2 k T}\right)=1 .
$$

The curve of critical values of nearest neighbors $z\left(\frac{k T}{J}\right)$ as the inverse function of exchange integral per electron in $k T$ units is shown at the Fig. 2.

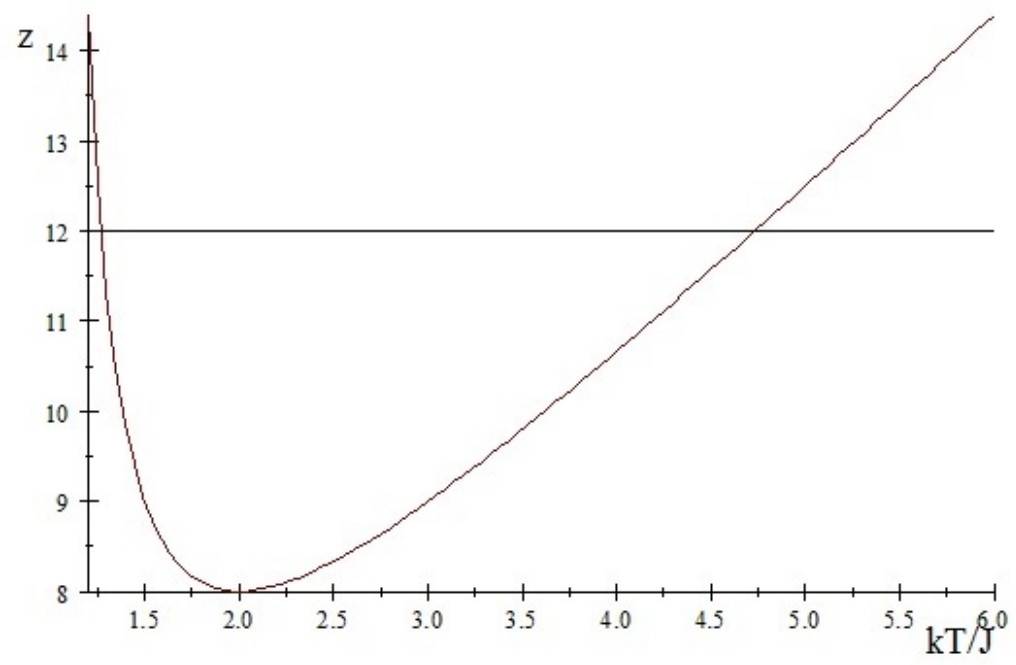

FIG. 2. The plot of the function $z=\frac{2 x^{2}}{x-1}, x=\frac{k T}{J}$. The straight horizontal line marks $z=12$.

The Boltzmann constant is marked as $k$ at the abscissa label

The solution of the quadratic equation $\left(1-\frac{\beta}{z}\right) \beta=2$ gives the roots:

$$
\beta_{1,2}=\frac{z}{2} \pm \sqrt{\frac{z^{2}}{4}-2 z}
$$

the minimal temperature yields for the case:

$$
\beta_{\max }=\frac{z J}{k T_{\min }}, \quad T_{\min }=\frac{z J}{k \beta_{\max }},
$$

and the maximum is equal to:

$$
\beta_{\min }=\frac{z J}{k T_{\max }}, \quad T_{\max }=\frac{z J}{k \beta_{\min }} .
$$

For the minimal case of $z=8$, (iron) a direct application of the theory is impossible, because of the ferromagnetic range is restricted by one point, look e.g. [5,6]. Outside the range, the magnetization curve shows the behavior typical for a paramagnetic material.

For the case, marked at the Fig. $2, z=12$, we obtain $\beta=6 \pm 2 \sqrt{3}$, hence the minimal temperature yields for the case:

and the maximum

$$
T_{\min }=\frac{12 J_{12}}{k(6+2 \sqrt{3})},
$$

$$
T_{\max }=\frac{12 J_{12}}{k(6-2 \sqrt{3})} .
$$


We would estimate the exchange integral from (15) for $N i$, having:

$$
J_{N i}=\frac{T_{\max N i} k(6-2 \sqrt{3})}{12} .
$$

Plugging the result into (16) we compute the critical temperature:

$$
T_{\text {min }}=\frac{12 J_{N i}}{k(6+2 \sqrt{3})},
$$

This case, valid for $\mathrm{Ni}, \mathrm{Co}$, is described in [5], where the curve of magnetization as function of temperature is shown and close to experiments of $[6,7]$.

Then, the hysteresis phenomenon exists at $z \geq 8$. For a simulation of the effect we take $\beta=10$, see Fig. 3 .

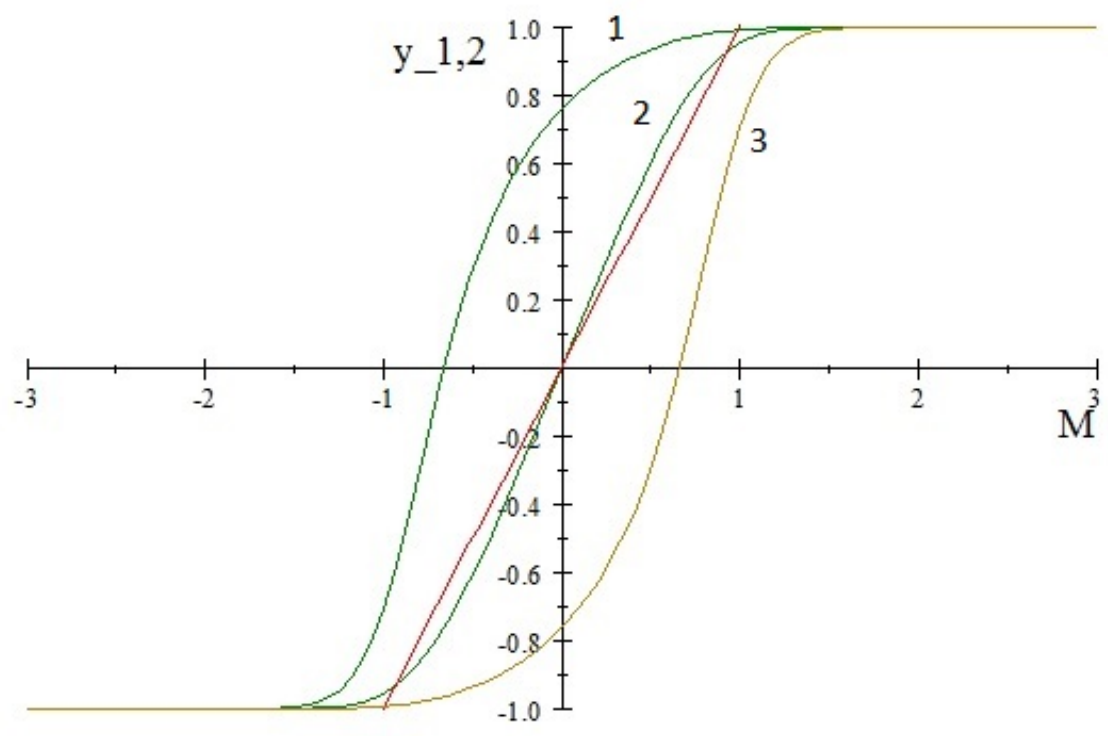

FIG. 3. The straight line (red) is $y_{1}=M$. The curves from upper to lower, numbers at the top: $y_{2}(M,-1)$ - green $-1, y_{2}(M, 0)-$ navy $-2, y_{2}(M, 1)-$ sienna -3

The double intersection of the curves $y_{1}=M$ and $y_{2}(M, \alpha)$ are demonstrated by Fig. 4 .

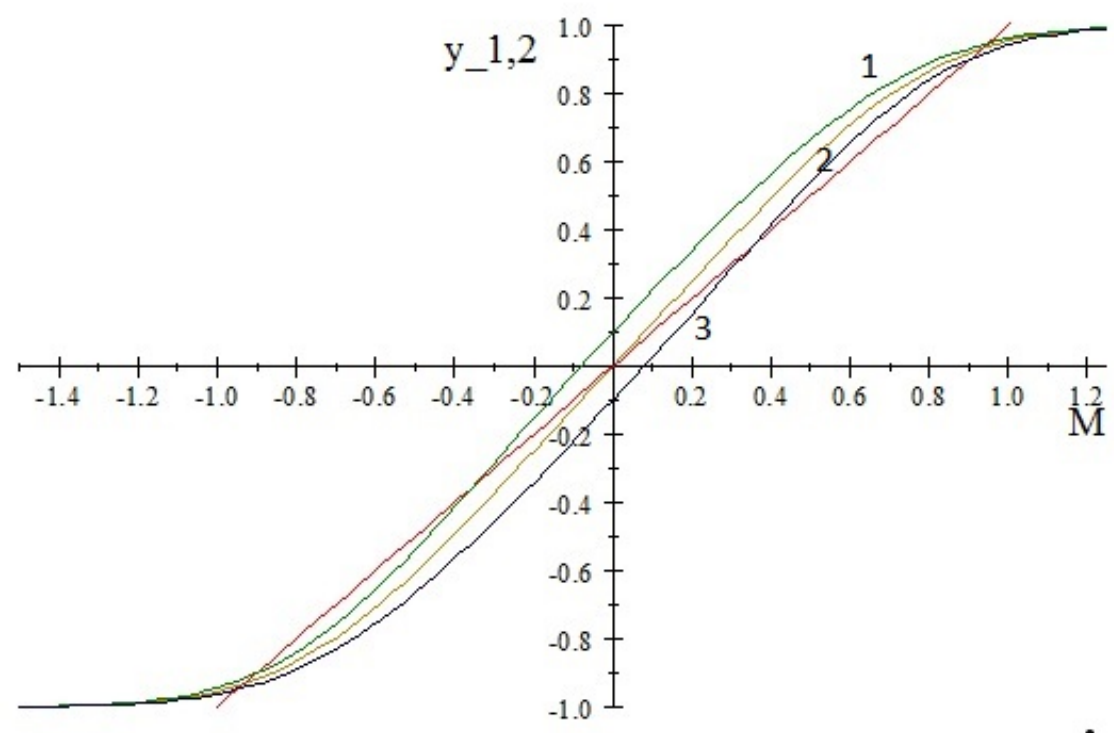

FIG. 4. The straight line (red) is $y_{1}=M$. The curves from upper to lower $y_{2}(M,-0.2)-$ green $1, y_{2}(M, 0)$ - navy $-2, y_{2}(M, 0.2)-$ sienna -3 
Generally, it is convenient to use the inverse to $M=\tanh \frac{x}{2}$ function:

$$
x=\ln \frac{1+M}{1-M}, \quad M \in(-1,1),
$$

which has double intersections with the curve (see (7))

$$
\omega=\alpha+\beta M-\beta^{2} \frac{M}{z}+\beta^{2} \frac{M^{3}}{2 z},
$$

giving the hysteresis curve, as it is shown at the Fig. 5.

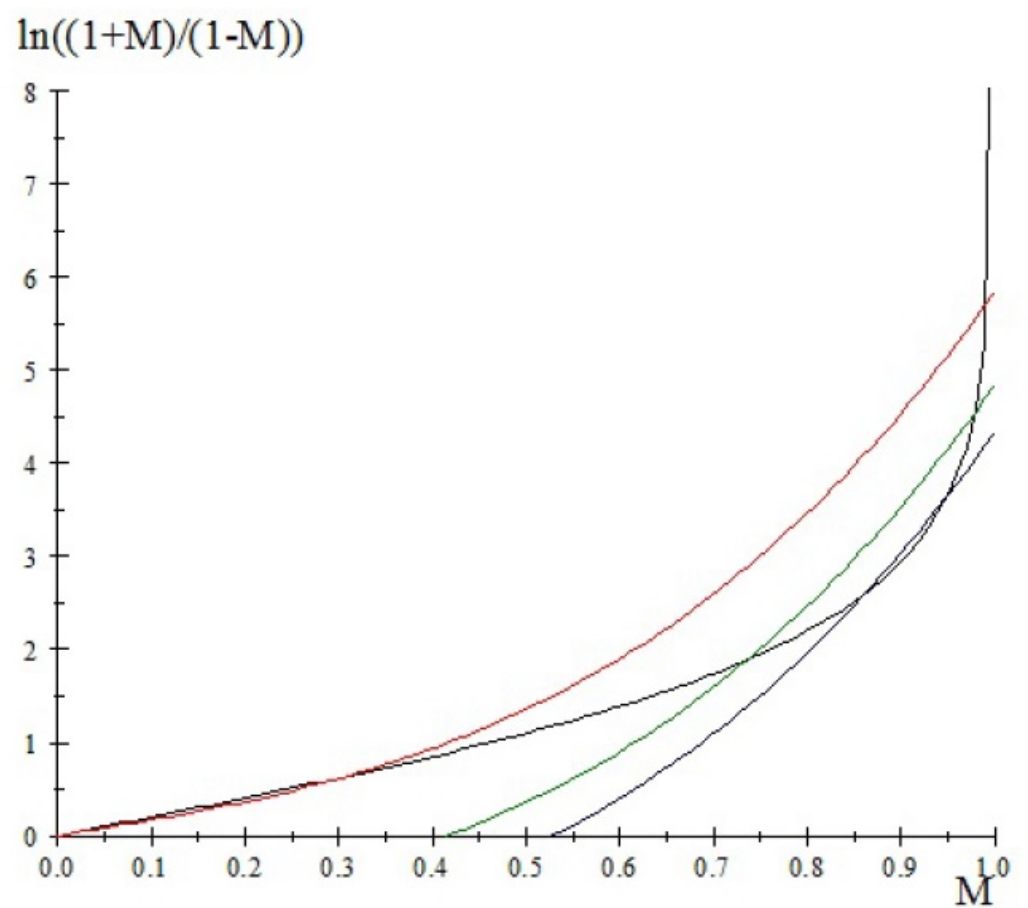

FIG. 5. The black curve shows the function marked at the ordinate. The colored curves shows the r.h.s. of the function (56) with $\beta=10, \alpha=0$ (red -1), $\alpha=-1$ (green - 2), $\alpha=-1.5$ (navy -3 ), numbers mark the curves

\section{Fine particles case}

\subsection{Back to partition function, surface-bulk division}

Figure 6 presents a nanoparticle cross-section schematically, that allows to estimate the number of atoms at the surface layer and under the surface (bulk) layers.

Let the nanoparticle radius be $N d$, with $N$ - number of layers and $d$ is the atom diameter, then the number of "surface" particles is estimated as:

$$
n_{a}=\frac{4}{3} \pi\left(N^{3}-(N-1)^{3}\right)=4 \pi\left(3 N^{2}-3 N+1\right),
$$

the number of "bulk" particles is equal to:

$$
n_{b}=\frac{4}{3} \pi(N-1)^{3} .
$$

Let $m$ be the spin projection quantum number. The partition function of states is built as in [1], taking as density of states the product of Gauss distribution and the distribution with mean energy for given spin s via evaluation for 


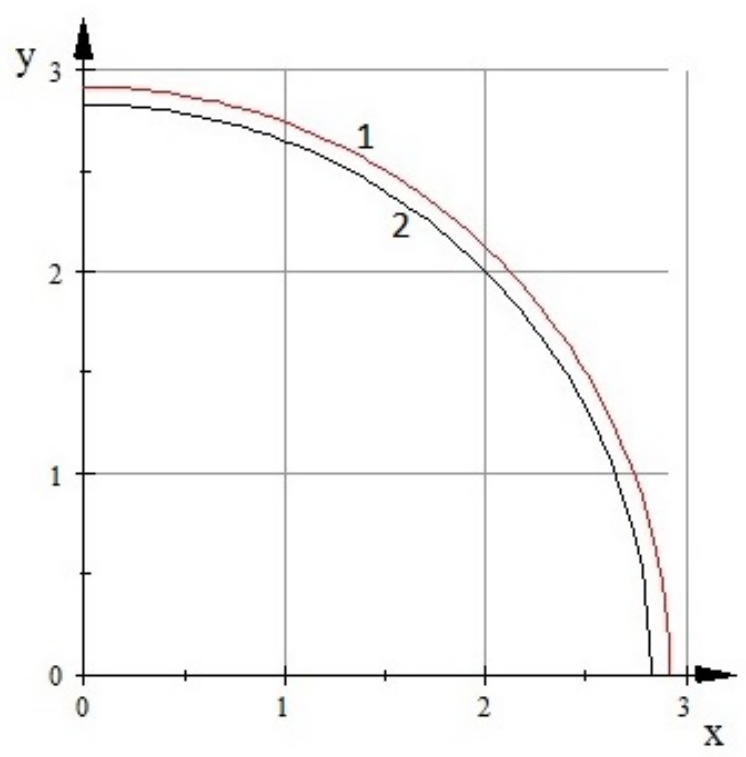

FIG. 6. The plot represents the cross-section of a nanoparticle, approximated by a sphere of radius $N d$, where $N$ is the number of layers and $d$ is the atom diameter. E.g. for $\mathrm{Ni}$ it is $d=1.49 \cdot 10^{-10}[\mathrm{~m}]$. The space between black curve (2) with radius $(N-1) d$ and the red curve (1) with radius $N d$ shows the surface monoatomic layer. Numbers of curves are in the parenthesis

irreducible representation ( $n_{\nu}$ its dimension) for electrons' permutations group; we keep the author's notations:

$$
\begin{array}{r}
\sum_{s=0, m=-s}^{n, s} \int_{-\infty}^{\infty} d \Delta E \frac{n_{\nu}}{\sqrt{2 \pi \overline{\left(\Delta E^{\nu}\right)^{2}}}} \exp \left\{\alpha m+\beta \frac{s^{2}}{2 n}-\frac{\Delta E}{k T}-\frac{\Delta E^{2}}{2 \overline{\left(\Delta E^{\nu}\right)^{2}}}\right\}= \\
\sum_{s=0}^{n} \sum_{m=-s}^{s} n_{\nu} \exp \left\{\alpha m+\beta \frac{s^{2}}{2 n}+\frac{\overline{\Delta\left(E^{\nu}\right)^{2}}}{2 k^{2} T^{2}}\right\} .
\end{array}
$$

After the principal bulk model simplification $J_{g}=J$ and some algebra we arrive at (4).

\subsection{Energy distribution for a tiny particle}

Now, let us take into account the difference between exchange integrals for surface and bulk atoms. Such division is stressed in [18]. We take, that the values of $J_{a, b}$ are the same for pairs bulk-bulk $\left(J_{b}\right)$; while surface-surface; and bulk-surface are denoted as $J_{a}$.The energy $E^{\nu}$ and the Gauss distribution parameter $\Delta E^{\nu}$, that is also expressed in terms of the symmetry group characters [3]. The result of Heitler for the energy is expressed as the sum by n-particle permutation group $g \in P[4]$, the exchange integrals are marked by group elements:

$$
E^{\nu}=\frac{1}{n_{\nu}} \sum_{g \in P} \chi^{\nu}(g) J_{g}
$$

Here, $\chi^{\nu}(g)=D_{i i}^{\nu}(g)$ is the character of the symmetry group irreducible representation (number $\nu$, the representation dimension is $n_{\nu}$ ), that numerates the energy terms of multielectron system. The characters of the permutation group are listed in [4], corrected in [1].

For the case with absence of space symmetry, as e.g. for amorphous matter, one has only the mentioned surfacebulk division of exchange integrals and the subgroups $G_{a} \in P$ that contains only permutations between electrons of surface-surface and bulk-surface atoms, while $G_{b} \in P$ contains the permutations only between bulk-bulk atoms, electrons. Both groups are the permutation ones of the corresponding orders. For clarity, we left only two different exchange integrals, marked by indices $a, b ; b$ for bulk and $a$ for surface. Then the mean energy reads:

$$
E^{\nu, \nu^{\prime}}=\frac{J_{a}}{n_{\nu^{\prime}}} \sum_{g \in G_{a}} \chi^{\nu^{\prime}}(g)+\frac{J_{b}}{n_{\nu}} \sum_{g^{\prime} \in G_{b}} \chi^{\nu}\left(g^{\prime}\right),
$$


we keep the number $\nu$ for the irreducible representation of the "bulk" subgroup $G_{b} \in P$. To explain the modification of the statistical sum with the surface-bulk subdivision we return to its origin. The sum by Heisenberg includes the terms with an energy and its Gauss distribution parameter, i.e. the mean square deviation of energy distribution.

The $\sum \Delta E_{n}=0$, as well as due to $\chi^{\nu}(e)=n_{\nu}$, again in terms of the characters, we derive, finally the mean square deviation from the mean value $E^{\nu}$ with more details compared with one from [3]:

$$
\overline{\left(\Delta E_{n}\right)^{2}}=\frac{J_{a}^{2}}{n_{\nu^{\prime}}^{2}} \sum_{g^{\prime}, g \in G_{a}}\left(\chi^{\nu^{\prime}}\left(g g^{\prime}\right)-\chi^{\nu^{\prime}}(g) \chi^{\nu^{\prime}}\left(g^{\prime}\right)\right)+\frac{J_{b}^{2}}{n_{\nu}^{2}} \sum_{g^{\prime}, g \in G_{b}}\left(\chi^{\nu}\left(g g^{\prime}\right)-\chi^{\nu}(g) \chi^{\nu}\left(g^{\prime}\right)\right),
$$

where the sums run the subgroups of the complete permutation group.

The energy (23) and the distribution parameter (24) enter the partition function exponent (21) linearly, hence the exponential property admits that the statistical may be factorized as in (3).

\subsection{Partition functions for a tiny particle}

The modification of he whole construction could be similarly done for $Z^{a}$ and $Z^{b}$ partition functions:

$$
\begin{gathered}
M^{a+b}=\frac{\partial \ln \left(Z_{a} Z_{b}\right)}{\partial \alpha}=\frac{\partial \ln \left(Z_{a}\right)}{\partial \alpha}+\frac{\partial \ln \left(Z_{b}\right)}{\partial \alpha}= \\
\frac{\partial \ln \left(\left[2 \cosh \frac{\omega_{a}}{2}\right]^{2 n_{a}}\right)}{\partial \alpha}+\frac{\partial \ln \left(\left[2 \cosh \frac{\omega_{b}}{2}\right]^{2 n_{b}}\right)}{\partial \alpha}= \\
\frac{n_{a}}{n} \tanh \frac{\omega_{a}}{2}+\frac{n_{b}}{n} \tanh \frac{\omega_{b}}{2} .
\end{gathered}
$$

where $n_{a}$ is given by (19) and $n_{b}$ by (20) correspondingly, so that:

$$
\frac{n_{a}}{n}=\frac{4 \pi\left(3 N^{2}-3 N+1\right)}{4 \pi N^{3}}=\frac{3 N^{2}-3 N+1}{N^{3}},
$$

similarly, the relative number of "bulk" particles is equal to:

Look the plots at Fig. 7

$$
\frac{n_{b}}{n}=\frac{(N-1)^{3}}{N^{3}}
$$

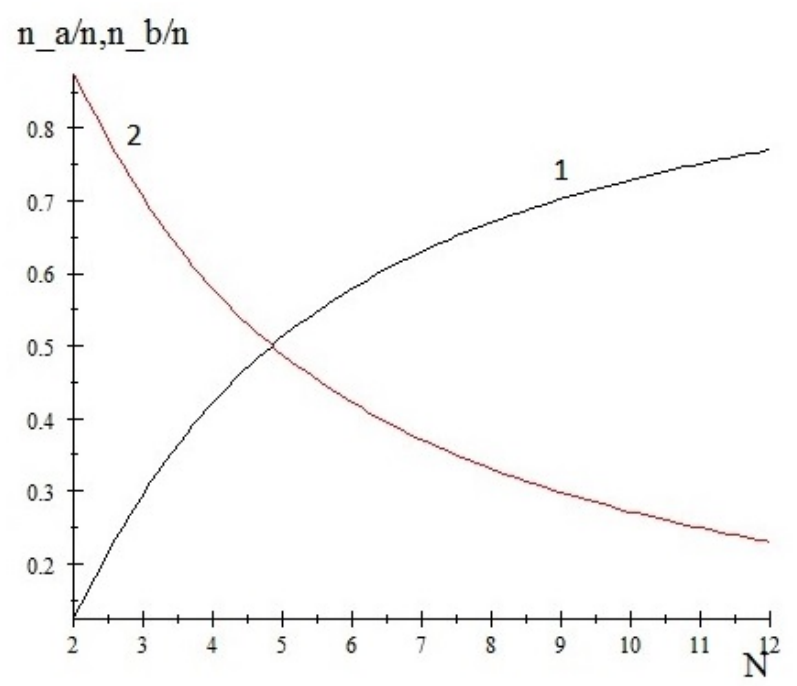

FIG. 7. The relative number of atoms in bulk (black - 1) and surface (red - 2) layers as the function of atomic layers $\mathrm{N}$

$M^{a+b}=M_{a}+M_{b}$ is a most probable value of magnetic quantum number projection per electron for a joint bulk and a surface parts. The functions $\omega_{a, b}$ are described by:

$$
\omega_{a}=\alpha+\beta_{a} M_{a}-\beta_{a}^{2} \frac{M_{a}}{z_{a}}\left(1-\frac{M_{a}^{2}}{2}\right) .
$$

similarily for $b$ case. The parameters $\beta_{a, b}=\frac{z_{a, b} J_{a, b}}{k T}$ depend on exchange integrals. The bulk one may be defined via Curie point temperature. 


\subsection{Numerical experiments}

For the numeric estimations let us choose the number of layers $N=5$ for $N i$ nanoparticle. For such layers number we have equal number of atoms. The parameters we have chosen are the following: the bulk exchange integral, evaluated via Curie temperature value $\left(\theta_{N i}=627 K\right) J_{N i}=1.8 \cdot 10^{-21} J$ [25]. The parameter $\beta_{b}=\frac{12 J_{N i}}{k_{B} T}=2$ for $T=795 K$ outside the ferromagnetic range. For the surface condition we take $z=8, \beta_{a}=\frac{8 \cdot 1,15 J_{N i}}{k_{B} T}=1.53$; the exchange integral corrected up $15 \%$, for the minor distance between the surface and bulk atoms.

The magnetization of the surface layer is presented at Fig. 8 . The corresponding bulk magnetization is superposed at the Fig. 9.

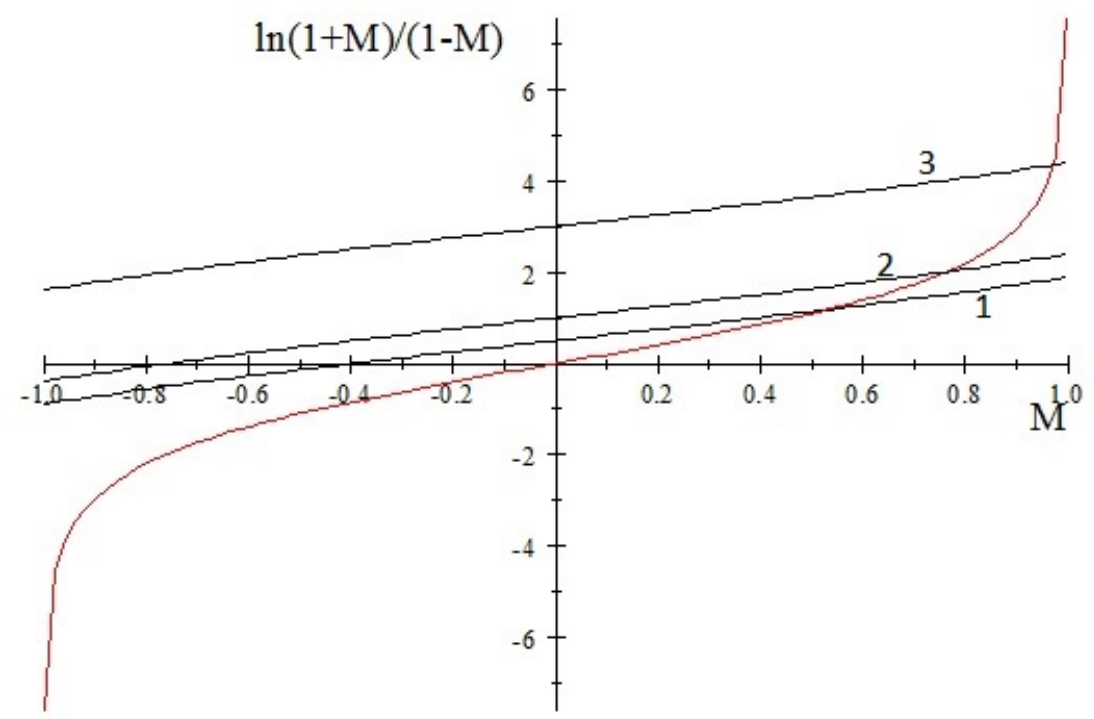

FIG. 8. The surface layer contribution to magnetization of a nanoparticle model. The three points of intersections of the inverse tanh function (red line) and $\omega_{a}$ black lines are shown for $\alpha=0.5,1.0,3.0$ growth up $(0.5-1,0.3-2)$. The last point $\alpha=3$ (3) stands close to magnetization saturation

\section{Electric field action}

\subsection{Polarisation, Stark effect}

A theory of perturbation of atoms by the external electric field $\vec{E}$ is based on the perturbed Fockian action [3]:

$$
H \phi=\left(H_{F}+V\right) \phi .
$$

There are two possibilities for the field account: if a medium unit, e.g. - molecule, has constant dipole momentum $\vec{p}=e \vec{d}$, where $V=e E\left(x+d_{x}\right), \vec{E}$ applied along $x$, reads as the conventional expansion by small parameter $\epsilon$, $\epsilon=e E$ :

$$
\phi=\phi_{0}+\epsilon \phi^{(1)}+\ldots
$$

In the first order it gives the following expression for an eigen function perturbation, evaluated as sum by $m \neq n$

$$
\phi_{p}^{(1)}=\sum_{m}^{\prime} \frac{x_{m p}+\left(d_{x}\right)_{m p}}{E_{m}-E_{p}} \phi_{0 m}
$$

where $x_{m n}$ are matrix elements of the Cartesian coordinate $x$ and $\left(d_{x}\right)_{m n}$ are proportional to the matrix elements projection of the constant dipole momentum $\vec{p} / e$ in non-perturbed states, between the eigenfunctions $\phi_{0 m}$ of the Fockian $H_{F}$. Note, that a stability of similar perturbations is studied in [26]. 


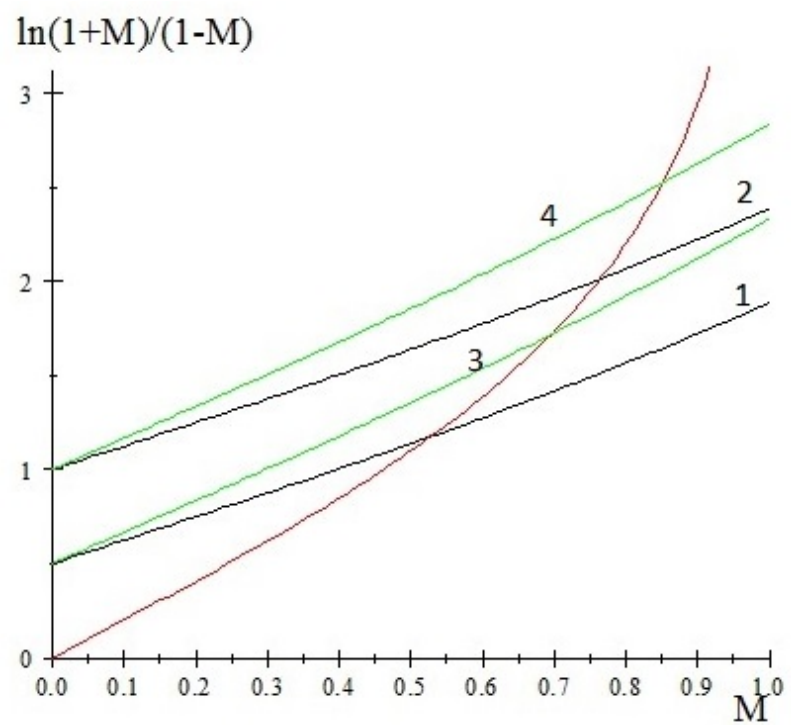

FIG. 9. The superposed layers contribution to magnetization of a nanoparticle model. The points of intersections of the inverse tanh function (red line) and $\omega_{a, b}$ black lines $-1,2$ for surface and green 3,4 for bulk are shown for $\alpha=0.5,1.0$. For $\alpha=0.5$, the curves 1,3 . The sum of magnetization contributions of the same order is quite visible with both values of $\alpha$ with the natural bulk prevails

\subsection{Exchange integrals perturbation}

Plugging (32) into exchange integrals $J_{i k}$, defined for the electrons coupled to the centers $i, k$, by the expression:

$$
J_{i k}=\frac{e^{2}}{2} \iint \frac{\rho_{i k}\left(\vec{r}, \vec{r}^{\prime}\right)}{\left|\vec{r}-\vec{r}^{\prime}\right|} d \vec{r} d \vec{r}^{\prime},
$$

where, in a spirit of he Fock paper [27], it may include all interacting electrons of the closest neighbors, as equal ones, hence indices omitted, reproducing [3]:

$$
\begin{gathered}
\rho\left(\vec{r}, \vec{r}^{\prime}\right)=\sum_{p} \phi_{p}^{*}(\vec{r}) \phi_{p}\left(\vec{r}^{\prime}\right)=\sum_{p}\left(\phi_{0 p}^{*}(\vec{r})+\epsilon \phi_{p}^{(1) *}(\vec{r})\right)\left(\phi_{0 p}\left(\vec{r}^{\prime}\right)+\epsilon \phi_{p}^{(1)}\left(\vec{r}^{\prime}\right)\right)= \\
\sum_{p} \phi_{0 p}^{*}(\vec{r}) \phi_{0 p}\left(\vec{r}^{\prime}\right)+\epsilon \sum_{p}\left[\phi_{p}^{(1) *}(\vec{r}) \phi_{0 p}\left(\vec{r}^{\prime}\right)+\phi_{0 p}^{*}(\vec{r}) \phi_{p}^{(1)}\left(\vec{r}^{\prime}\right)\right]+o\left(\epsilon^{2}\right)=\rho_{0}\left(\vec{r}, \vec{r}^{\prime}\right)+\epsilon \rho_{1}\left(\vec{r}, \vec{r}^{\prime}\right)+\ldots
\end{gathered}
$$

The electric field perturbs the exchange integral as it is prescribed by (35) with:

$$
\begin{aligned}
& J_{0}=\frac{e^{2}}{2} \iint \frac{\rho_{0}\left(\vec{r}, \vec{r}^{\prime}\right)}{\left|\vec{r}-\vec{r}^{\prime}\right|} d \vec{r} d \vec{r}^{\prime}, \\
& J_{1}=\frac{e^{2}}{2} \iint \frac{\rho_{1}\left(\vec{r}, \vec{r}^{\prime}\right)}{\left|\vec{r}-\vec{r}^{\prime}\right|} d \vec{r} d \vec{r}^{\prime},
\end{aligned}
$$

whence:

More details for $J_{1}$ gives:

$$
\beta=\frac{z\left(J_{0}+\epsilon J_{1}\right)}{k T}=\beta_{0}+\epsilon \beta_{1}
$$

$$
J_{1}=\frac{e^{2}}{2} \sum_{p, m}^{\prime} \frac{\left(x_{m p}+\left(d_{x}\right)_{m p}\right) J_{m p}^{0}}{E_{m}-E_{p}},
$$

with the notation for $J_{p m}^{0}=\int \frac{\phi_{0 m}^{*}(\vec{r}) \phi_{0 p}\left(\vec{r}^{\prime}\right)}{\left|\vec{r}-\vec{r}^{\prime}\right|} d \vec{r} d \vec{r}^{\prime}$.

The matrix elements $J_{p m}^{0}$ are evaluated between the unperturbed Fockian eigenfunction, that yields (39). In the phenomenologic theory we consider $J_{0}$ and $J_{1}$ as parameters. 


\section{Magneto-electric effect}

\subsection{Material equation of state}

First-principles methods to study magnetoelectric phenomena are reviewed as follows. Plugging (38) into (4) yields:

$$
\frac{Z}{F}=\left[2 \cosh \left(\frac{\alpha+\beta_{0} M-\beta_{0}^{2} \frac{M}{z}+\beta_{0}^{2} \frac{M^{3}}{2 z}+\epsilon \beta_{1} M\left(1-\frac{2}{z} \beta_{0}+\frac{M^{2}}{2 z} \beta_{0}\right)+\epsilon^{2} \beta_{1}^{2}\left(\frac{M^{3}}{2 z}-\frac{M}{z}\right)}{2}\right)\right]^{2 n},
$$

being the base for the partition function expression with the electric field account.

The mean (most probable) value of the magnetization per electron is defined by the Heisenberg relation (7):

$$
M(\alpha, \epsilon)=\tanh \frac{\alpha+\left(1-\frac{\beta(\epsilon)}{z}\right) \beta(\epsilon) M+\beta(\epsilon)^{2} \frac{M^{3}}{2 z}}{2},
$$

that now includes not only magnetic field $H$ as the parameter, but also the electric field $E$ via $\epsilon$. It constitutes the magnetic material relation in implicit form.

Let us illustrate the solution of the transcendent equation (41) by the Fig. 10. It shows that there is a minimal value of $\epsilon$ for a solution existence.

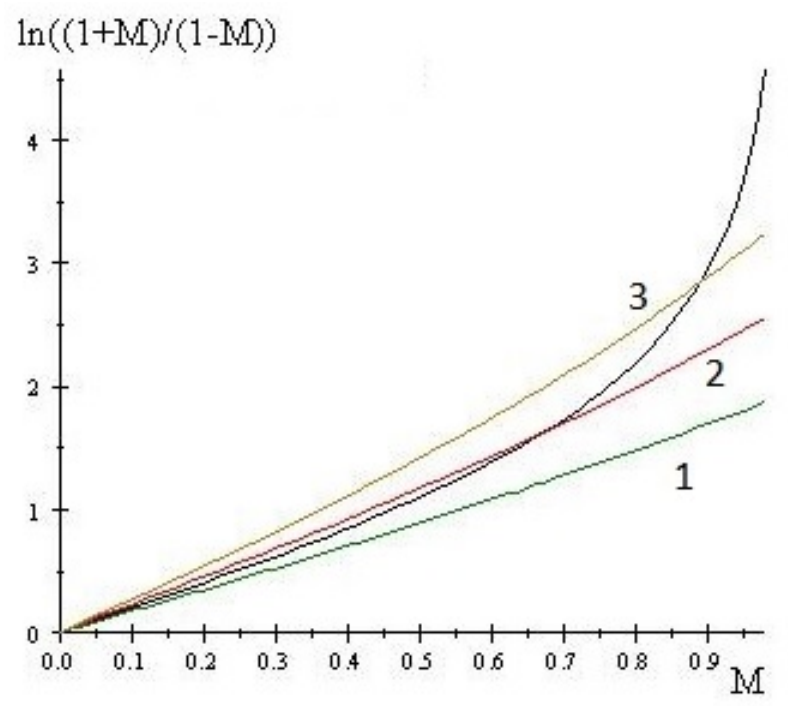

FIG. 10. The points of intersections of the inverse tanh function (black line) and $\omega(M)$, green line 1 for $\epsilon=0.1$, the red one -2 for $\epsilon=1$ and the sienna -3 for $\epsilon=2$ are shown for $\alpha=0$. The case of $\epsilon=0.1$ is out of a solution range

The derivative of $\ln Z$ by $\epsilon$ gives the polarization on electron as:

$$
P=\frac{p_{0}}{n}=e \frac{\partial \ln Z}{n \partial \epsilon}=e \frac{\partial \omega}{\partial \epsilon} \tanh \frac{\omega}{2}, \quad \frac{\partial \omega}{\partial \epsilon}=\beta_{1} M-2 \beta \frac{\beta_{1}}{z} M+2 \beta \beta_{1} \frac{M^{3}}{2 z}+\beta\left[\left(1-\frac{\beta}{z}\right)+\frac{3 M^{2}}{2 z} \beta\right] \frac{\partial M}{\partial \epsilon},
$$

or, in alternative form,

$P=e\left(\beta_{1} M\left(1-\frac{2}{z} \beta_{0}+\frac{M^{2}}{2 z} \beta_{0}\right)+2 \epsilon \beta_{1}^{2} \frac{M}{z}\left(\frac{1}{2} M^{2}-1\right)\right) \tanh \left(\frac{\alpha+\beta_{0} M-\beta_{0}^{2} \frac{M}{z}+\beta_{0}^{2} \frac{M^{3}}{2 z}}{2}+A\right)+e \frac{\partial \ln Z}{n \partial M} \frac{\partial M}{\partial \epsilon}$,

where

$$
A=\left(\frac{1}{2} \frac{M^{3}}{z} \beta_{1}^{2}-\frac{M}{z} \beta_{1}^{2}\right) \epsilon^{2}+\left(M \beta_{1}+\frac{M^{3}}{z} \beta_{0} \beta_{1}-2 \frac{M}{z} \beta_{0} \beta_{1}\right) \epsilon .
$$

The second term in (42), $\frac{\partial M}{\partial \epsilon}$ should be evaluated along the solution of (41) path. The condition of a solution existence repeats the inequality (8) but now it should be solved with respect to $\epsilon$, or for the boundary,

$$
-\frac{1}{z}\left(\beta_{0}+\epsilon \beta_{1}\right)\left(\beta_{0}-z+\epsilon \beta_{1}\right)=\beta_{0}-\frac{1}{z} \beta_{0}^{2}+\epsilon \beta_{1}-\frac{1}{z} \epsilon^{2} \beta_{1}^{2}-\frac{2}{z} \epsilon \beta_{0} \beta_{1}=2 .
$$


The range of interest lies between the roots of this equations:

$$
-\frac{1}{2 \beta_{1}}\left(-z+2 \beta_{0}+z \sqrt{\frac{1}{z}(z-8)}\right), \quad \frac{1}{2 \beta_{1}}\left(z-2 \beta_{0}+z \sqrt{\frac{1}{z}(z-8)}\right),
$$

for $z=12$ it gives $\frac{1}{2 \beta_{1}}\left(4 \sqrt{3}-2 \beta_{0}+12\right),-\frac{1}{2 \beta_{1}}\left(2 \beta_{0}+4 \sqrt{3}-12\right)$.

To evaluate the derivative $\frac{\partial M}{\partial \epsilon}$, we use the approximate expansion of the both sides of (41), as is demonstrated by the plot Fig. 11. Algebraically it appears as:

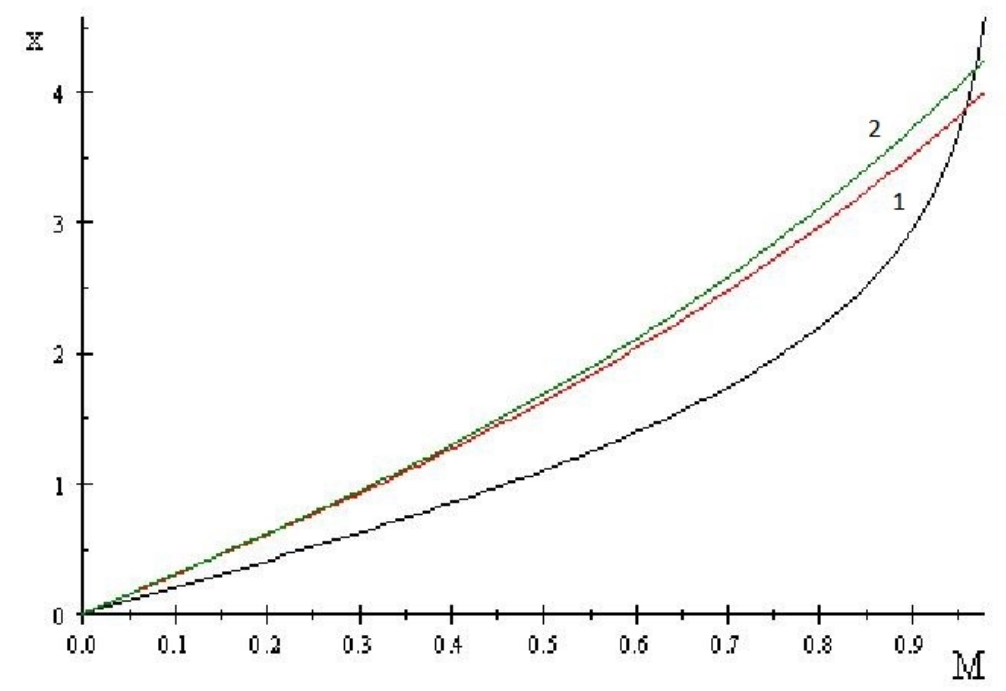

FIG. 11. The small variation of a solution of (41) by the change of $\beta$, for $z=12, \beta_{0}=5.3$, red $1, \epsilon \beta_{1}=0.3, \beta=5.6$ - green -2

for the r.h.s. $\alpha+(\beta+x)(M+y)-(\beta+x)^{2} \frac{M+y}{z}+(\beta+x)^{2} \frac{(M+y)^{3}}{2 z}$ and for the left one,

$$
\ln \frac{1+(M+y)}{1-(M+y)}=\ln \left(-\frac{1}{M-1}(M+1)\right)-y\left(\frac{1}{(M-1)^{2}}(M+1)-\frac{1}{M-1}\right) \frac{M-1}{M+1}+O\left(y^{2}\right) .
$$

The linear in $y$ term is equal to:

$$
\frac{1}{2} y \frac{4 z-5 M^{2} \beta^{2}+3 M^{4} \beta^{2}-2 z \beta+2 \beta^{2}+2 M^{2} z \beta}{z(M-1)(M+1)},
$$

It contains the factor $4 z-5 M^{2} \beta^{2}+3 M^{4} \beta^{2}-2 z \beta+2 \beta^{2}+2 M^{2} z \beta$, that can be zero when:

$$
M_{r}^{2}= \pm \frac{1}{6 \beta}\left(5 \beta+\sqrt{\beta^{2}+48 \beta}-24\right) \text {. }
$$

In such points a solution of the equation for $y$ does not exist. Hence, far enough of these points, equalizing the linear in $x, y$ parts with $\alpha=0$, we arrive at:

$$
\frac{\partial M}{\partial \epsilon} \approx \frac{y}{x}=\frac{2 M\left(z-2 \beta+M^{2} \beta\right)(M-1)(M+1)}{4 z-5 M^{2} \beta^{2}+3 M^{4} \beta^{2}-2 z \beta+2 \beta^{2}+2 M^{2} z \beta} .
$$

Plugging it into (43) yields:

$$
\frac{\partial \omega}{\partial \epsilon}=\beta_{1} M-2 \beta \frac{\beta_{1}}{z} M+2 \beta \beta_{1} \frac{M^{3}}{2 z}+\beta\left[\left(1-\frac{\beta}{z}\right)+\frac{3 M^{2}}{2 z} \beta\right] \frac{2 M\left(z-2 \beta+M^{2} \beta\right)(M-1)(M+1)}{4 z-5 M^{2} \beta^{2}+3 M^{4} \beta^{2}-2 z \beta+2 \beta^{2}+2 M^{2} z \beta} .
$$

That gives explicit form for the polarization $P(E)$ as function of electric field, see the Fig. 12.

If to write the material relations in approximation of Taylor expansion of both $P, M$ in $\alpha \epsilon$ plane up to the first order, we arrive at:

$$
P=P_{0}+\epsilon P_{1}+\alpha P^{1}, \quad M=M_{0}+\alpha M_{1}+\epsilon M^{1},
$$


where:

$$
\begin{gathered}
P_{0}=\left.e \frac{\partial \omega}{\partial \epsilon} \tanh \frac{\omega}{2}\right|_{\epsilon, \alpha=0}=e\left(\beta_{1} M-2 \beta_{0} \frac{\beta_{1}}{z} M+2 \beta_{0} \beta_{1} \frac{M^{3}}{2 z}+\left.\beta_{0}\left[\left(1-\frac{\beta_{0}}{z}\right)+\frac{3 M^{2}}{2 z} \beta_{0}\right] \frac{\partial M}{\partial \epsilon}\right|_{\epsilon=0}\right) \mathcal{T} \\
P_{1}=\left.\frac{\partial P}{\partial \epsilon}\right|_{\alpha, \epsilon=0}=\left.e\left[\frac{\partial^{2} \omega}{\partial \epsilon^{2}} \tanh \frac{\omega}{2}+\left(\frac{\partial \omega}{\partial \epsilon}\right)^{2} \frac{1}{2}\left(1-\mathcal{T}^{2}\right)\right]\right|_{\epsilon=0}, \quad P^{1}=\left.\frac{\partial P}{\partial \alpha}\right|_{\alpha, \epsilon=0}=\frac{e}{2} \frac{\partial \omega}{\partial \epsilon}\left(1-\mathcal{T}^{2}\right) \\
M_{0}=\mathcal{T}, \quad M_{1}=\frac{1}{2}\left(1-\mathcal{T}^{2}\right), \quad M^{1}=\left.\frac{\partial M}{\partial \epsilon}\right|_{\alpha, \epsilon=0},
\end{gathered}
$$

with $\mathcal{T}=\tanh \frac{\beta_{0} M}{4 z}\left(M^{2} \beta_{0}-2 \beta_{0}+2 z\right) . \quad u=-\frac{1}{2} M^{2} \beta_{1}^{2} \frac{\left(z-2 \beta_{0}+M^{2} \beta_{0}\right)^{2}}{z^{2}}, v=-\frac{M}{z} \beta_{1}^{2}\left(2-M^{2}\right)$, that demonstrates magneto-electric effects as in [28].

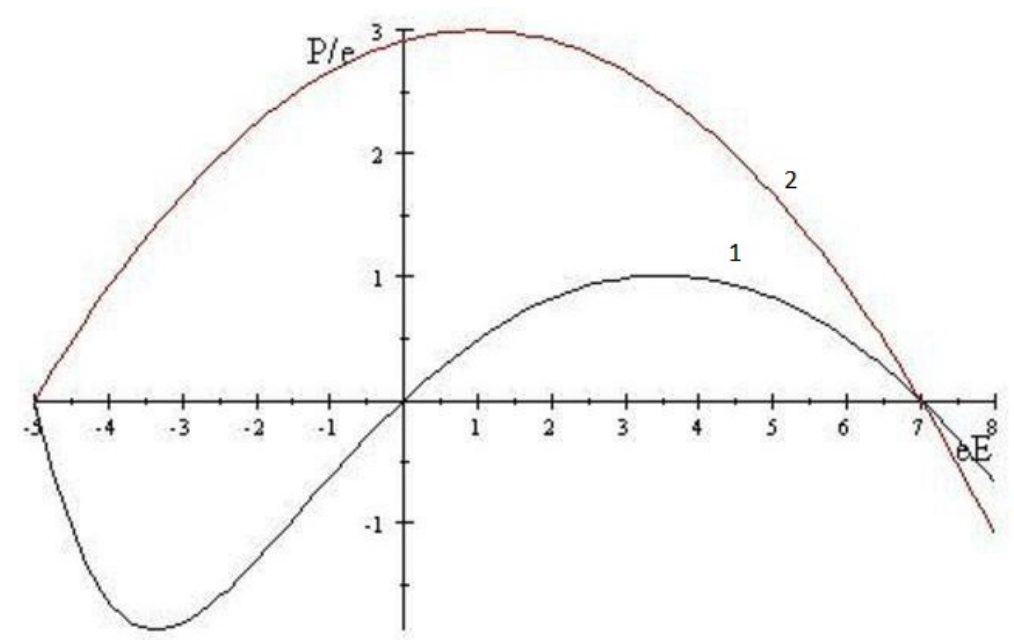

FIG. 12. The dependence the polarization $P$ on field $E$ - curve 1 . The range of a solution of (41) existence lies between the red parabola (curve 2) roots, for $z=12, \beta_{0}=5, \beta_{1}=1$

As we see, the argument of the hyperbolic tangent function in the r.h.s. of (42), contains the quadratic polynomial in the electric field $\epsilon$. We observe the nonlinear dependence of $P(\epsilon)$. The key cross-terms of the polarization $P^{1}$ and magnetization $M^{1}$ material equations expansion coefficients in (49) are shown at the Fig. 13 as function of the background magnetization.

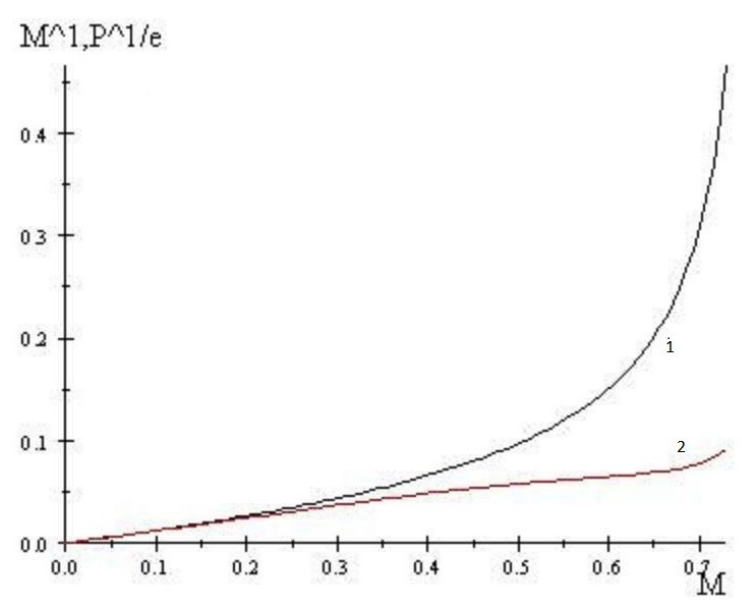

FIG. 13. The $M^{1}(M)$ - black - 1 and $P^{1}(M)$ - red - 2 are plotted within the range restricted by (45) 


\subsection{Magnetoelectric phenomena for nanoparticles}

The consideration of magnetoelectric effects may be done along the lines of the Sec. 3.3. We base on the equation (25) while the functions (56) contain the parameters, $\beta_{a, b}$, "splitted" by the electric field action as, for example $\beta_{a}=\beta_{a}^{0}+\epsilon \beta_{a}^{1}$ in direct analogy with (38). So, the basic equations for magnetization and polarization are

$$
\begin{gathered}
M^{a+b}=\frac{\partial \ln \left(Z_{a}\right)}{\partial \alpha}+\frac{\partial \ln \left(Z_{b}\right)}{\partial \alpha}= \\
\frac{\partial \ln \left(\left[2 \cosh \frac{\omega_{a}^{e}}{2}\right]^{2 n_{a}}\right)}{\partial \alpha}+\frac{\partial \ln \left(\left[2 \cosh \frac{\omega_{b}^{e}}{2}\right]^{2 n_{b}}\right)}{\partial \alpha}= \\
\frac{n_{a}}{n} \tanh \frac{\omega_{a}^{e}}{2}+\frac{n_{b}}{n} \tanh \frac{\omega_{b}^{e}}{2},
\end{gathered}
$$

and, for the polarization:

$$
\begin{gathered}
P^{a+b}=e \frac{\partial \ln \left(Z_{a}\right)}{\partial \epsilon}+e \frac{\partial \ln \left(Z_{b}\right)}{\partial \epsilon}= \\
e \frac{\partial \ln \left(\left[2 \cosh \frac{\omega_{a}^{e}}{2}\right]^{2 n_{a}}\right)}{\partial \epsilon}+e \frac{\partial \ln \left(\left[2 \cosh \frac{\omega_{b}^{e}}{2}\right]^{2 n_{b}}\right)}{\partial \epsilon}= \\
e \frac{n_{a}}{n} \frac{\partial \omega_{a}^{e}}{\partial \epsilon} \tanh \frac{\omega_{a}^{e}}{2}+e \frac{n_{b}}{n} \frac{\partial \omega_{b}^{e}}{\partial \epsilon} \tanh \frac{\omega_{b}^{e}}{2} .
\end{gathered}
$$

where, plugging $\beta_{a}=\beta_{a}^{0}+\epsilon \beta_{a}^{1}$, we write:

$$
\omega_{a}^{e}=\alpha+\left(\beta_{a}^{0}+\epsilon \beta_{a}^{1}\right) M_{a}\left(1-\beta_{a} \frac{\left(1-\frac{M_{a}^{2}}{2}\right)}{z_{a}}\right),
$$

and, quite similar for $\omega_{b}^{e}$.

Let us plot the scheme of the equations (50) solution, again for the case, as for the Nickel, for magnetic properties see [29]. Taking the same values for the bulk contribution as at Fig. 14, and for the surface as in the Table 1:

TABLE 1. Nanoparticle parameters

\begin{tabular}{|c||c|c|c|}
\hline Atom place & $\beta^{0}$ & $\beta^{1}$ & $\mathrm{z}$ \\
\hline \hline surface(a) & 1.53 & 1 & 8 \\
\hline bulk(b) & 2 & 1 & 12 \\
\hline
\end{tabular}

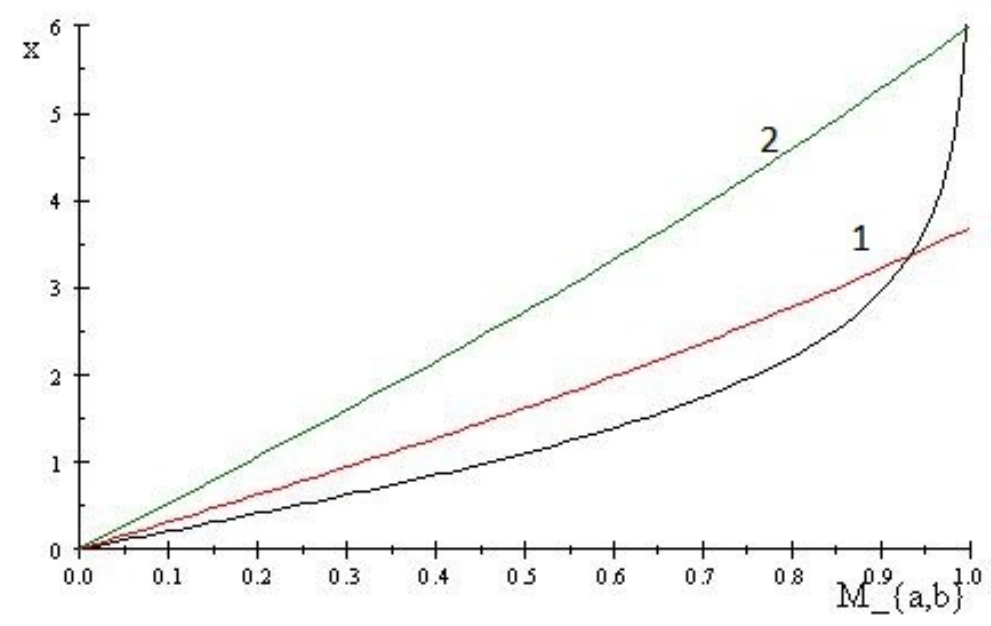

FIG. 14. The superposed layers contribution to magnetisation of a nanoparticle in the field with $\epsilon=1$. The points of intersections of the inverse tanh function (black line) $x=\ln \frac{1+M}{1-M}$ and $\omega_{a, b}$ red -1 line for surface and green -2 for bulk are shown for $\alpha=0$. The sum of magnetization contributions of the same order is quite visible with both values of $\beta_{a}, z_{a}$ and $\beta_{b}, z_{b}$ with the natural bulk prevails 


\section{Conclusion}

We do understand, that the theory has model restrictions that are discussed in the Heisenberg papers, that do not allow literal application of the model, for example to Fe-based magnetics.

However, the use of a generalized Heisenberg theory allows one to classify matters in respect to matter relations and existence of ferro- properties of nanoparticles at least for the number of closest neighbors $z>8$. It also allows one to construct the hysteresis curves for given values of the structure and electron states parameters. Modifications for nanotubes naturally introduce the closest neighbors exchange integrals defined by the nano-object symmetry and position of them in bulk or surface of it. This gives a model thermodynamic description on the basis of the Heisenberg model of the partition function, which allows natural modifications.

\section{Acknowledgements}

The author acknowledges discussions with V. Rodionova.

\section{References}

[1] Heisenberg W. Zur Theorie des Ferromagnetismus. Zs. Phys., 1928, 49, P. 619-636. Zur Quantentheorie des Ferromagnetismus. Probleme der Modernen Physik, A. Sommerfeld Festschrift, Leipzig, 1928, P. 114-122.

[2] Vázquez M. Magnetic Nano- and Microwires: Design, Synthesis, Properties and Applications. Woodhead Publishing Series in Electronic and Optical Materials. Elsevier Science, 2015, 314 p.

[3] Leble S. Heisenberg chain equations in terms of Fockian covariance with electric field account and multiferroics in nanoscale. Nanosystems: Physics, Chemistry, Mathematics, 2019, 10(1), P. 18-30.

[4] Heitler W. Stäorungsenergie und Austausch beim Mehrkäorperproblem. Zs. Phys. 1927, 46, P. 47. Zur Gruppentheorie der hom'oopolaren chemischen Bindung, 1928, 835 p.

[5] Inglis D.R. The Heisenberg Theory of Ferromagnetism. Phys. Rev., 1932, 42, P. 442.

[6] Bitter F. On the Interpretation of Some Ferromagnetic Phenomena, Phys. Rev, 1932, 39, P. 337-345.

[7] Tyler F. The magnetization-temperature curves of iron, cobalt and nickel. Phil. Mag., 1931, 11, P. 596.

[8] Coey J.M.D. Magnetism and magnetic materials. Cambridge University Press, Cambridge, 2009.

[9] Aharoni A. Introduction to the Theory of Ferromagnetism, Clarendon Press, Oxford, 1996, $192 \mathrm{p}$.

[10] Nakano F. The Heisenberg Theory of Ferromagnetism of the Crystal constituted by the Atoms with Spin One. Progress of Theoretical Physics, 1953, 9(4), P. 403-413.

[11] Heisenberg W. Zur Theorie des Magnetpstriktin und der Magnetisierungkurve. Zs. Phys., 1931, 69, P. $287-297$.

[12] Singh N.The story of magnetism: from Heisenberg, Slater, and Stoner to Van Vleck, and the issues of exchange and correlation. arXiv:1807.11291 [cond-mat.str-el], 2018.

[13] Borovik E.S., Eremenko V.V., Milner A.S. Lectures on magnetism. 3-rd ed. Revised. and add. M.: Fizmatlit, 2005. (In Russian)

[14] Lakshmanan M. The fascinating world of the Landau-Lifshitz-Gilbert equation: an overview. Phil. Trans. R. Soc. A, 2011, 369, P. 1280-1300.

[15] Schollwöck U., Richter J., Farnell D.J.J., Bishop R.F. Quantum Magnetism, Lecture Notes in Physics, 645, Springer-Verlag, Berlin, 2004, $381 \mathrm{p}$.

[16] Martinez-Garcia J.C., Rivas M., Lago-Cachón D., Garcia J.A. First-order reversal curves analysis in nanocrystalline ribbons. J. Phys. D: Appl. Phys., 2014, 47, P. 015001.

[17] Martinez-Garcia J.C., Rivas M., Garcia J.A. Induced ferro-ferromagnetic exchange bias in nanocrystalline systems. Journal of Magnetism and Magnetic Materials, 2015, 377, P. 424.

[18] Klaus D. Sattler-Ed. Handbook of Nanophysics: Nanoparticles and Quantum Dots, CRC press, 2011.

[19] Gaikwad A.S., More S.S., et al. Int. Res. J. of Science and Engineering, 2018, A5, P. 41-44.

[20] Valasek J. Piezo-electric and allied phenomena in Rochelle salt. Phys. Rev., 1921, 17, P. 475-481.

[21] Spaldin, Nicola A., Fiebig, Manfred. The renaissance of magnetoelectric multiferroics. Science, 2005, 309(5733), P. 391-392.

[22] Birola T., Benedek N.A., et al. The magnetoelectric effect in transition metal oxides: Insights and the rational design of new materials from first principles Current Opinion. Solid State and Materials Science, 2012, 16(5), P. 227-242.

[23] Reichl L.E. A Modern Course in Statistical Physics, 2-nd Edition. A Wiley-interscience publication, John Wiley and Sons, inc., New York, Chichester, Weinheim, Brisbane, Singapore, Toronto, 1998, 822 p.

[24] Heisenberg W. Zur Quantentheorie des Ferromagnetismus. Probleme der Modernen Physik, A. Sommerfeld Festschrift, Leipzig, 1928, P. 114122.

[25] Jössang P., Jössang A.C., Pouillet S.M. member of the French Academy of Sciences, discovered the "Curie" point in ... 1832. Science Tribune, January 1997, URL: http: / / www.tribunes.com/tribune/art97/jos2e.htm.

[26] Popov I.Yu. Hydrodynamic stability and perturbation of the Schrodinger operator. Lett. Math. Phys., 1995, 35(2), P. 155-161.

[27] Fock V. An Approximate Method for Solving the Quantum Many-Body Problem. Zs. Phys., 1930, 61, P. 126-148; TOI 1931, 5, 51, 1; UFN 1967, 93, 2, 342.

[28] Dzyaloshinskii I.E. On the magneto-electrical effect in antiferromagnets. J. Exp. Theor. Phys., 1959, 37, P. 881-882.

[29] Nasirpouri F., Barzegar S., et al. Mesophase micelle-assisted electrodeposition and magnetisation behavior of meso-porous nickel films for efficient electrochemical energy and magnetic device applications. Applied Surface Science, 2019, 471, P. 776-785. 